\title{
Specific and Sensitive Fluorescence Anisotropy Sensing of Guanine- Quadruplex Structures via a Photoinduced Electron Transfer Mechanism
}

\author{
Dapeng Zhang, ${ }^{\dagger}$ Hujun Shen, ${ }^{\ddagger}$ Guohui Li, ${ }^{\ddagger}$ Bailin Zhao, ${ }^{\dagger}$ Anchi Yu, ${ }^{\perp}$ Qiang Zhao, ${ }^{\S}$ and Hailin Wang ${ }^{*}{ }^{\dagger}$ \\ ${ }^{\dagger}$ State Key Laboratory of Environmental Chemistry and Ecotoxicology, Research Center for Eco-Environmental Sciences, Chinese \\ Academy of Sciences, Beijing, 100085, P. R. China \\ ${ }^{\ddagger}$ State Key Laboratory of Molecular Reaction Dynamics, Dalian Institute of Chemical Physics, Chinese Academy of Sciences, Dalian, \\ 116023, P. R. China \\ ${ }^{\S}$ Research Center for Environmental Science and Engineering, Shanxi University, Taiyuan, Shanxi Province, 030006, P. R. China \\ ${ }^{\perp}$ Department of Chemistry, Renmin University of China, Beijing, 100872, P. R. China
}

Supporting Information

ABSTRACT: Fluorescence anisotropy (FA) is a homogeneous, ratiometric, and real-time analytical technology. By selective labeling of a guanine $(\mathrm{G})$-quadruplex motif with tetramethylrhodamine (TMR), here, it is established that a large reduction in FA response can be specifically associated with the unfolding $\rightarrow$ folding transition of G-quadruplex structures. On the basis of fluorescence intensity, polarization and lifetime analysis, and molecular docking simulation, the mechanism was found to be that the labeled fluorophore (TMR) can intramolecularly interact with adjacent $\mathrm{G}$ bases in an unfolded G-quadruplex motif, which allows for the photoinduced electron transfer (PET) occurring between the fluorophore and $\mathrm{G}$ bases, leading to a short

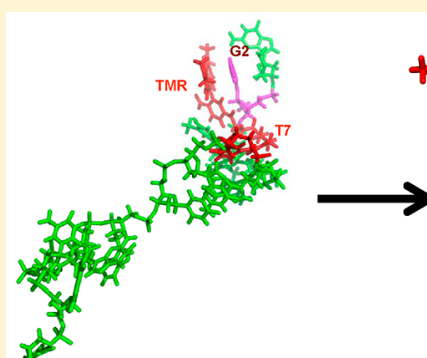

Slow rotation

High FA

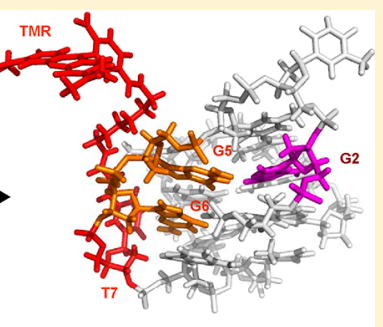

Fast rotation Low FA fluorescence lifetime. Upon the folding of the motif to form a stable G-quadruplex structure, the intramolecular interactions and the concomitant PET could be eliminated with an increased fluorescence lifetime, leading to a large reduction in the FA response. On the basis of this mechanism, a novel, specific, and sensitive FA approach was developed for the detection of biologically and functionally important G-quadruplex structures. The approach is examined and validated using one normal Gquadruplex motif, five mutants, and six small cations and is potentially applicable to the study of G-quadruplexes at single molecule level, ligand screening, profiling of highly ordered DNA nanostructures, and biosensing.

G uanine $(\mathrm{G})$-quadruplex motifs, occurring throughout the human genome, are involved in telomere maintenance and regulation of gene expression, ${ }^{1}$ through folding into Gquadruplexes as four-stranded helical structures held together by a guanine core, ${ }^{2}$ and are active targets for novel anticancer therapies. ${ }^{3,4}$ They are also found in many functional nucleic acids (aptamers, DNAzymes, and DNA nanostructures), in which the formation of G-quadruplex structures contributes greatly to their functions (specific recognition and catalytic activity). ${ }^{5-8}$ The exquisite structure of G-quadruplex can be directly characterized using nuclear magnetic resonance (NMR) spectroscopy, ${ }^{9,10}$ and X-ray crystallography. ${ }^{11}$ Although $\mathrm{X}$-ray crystallographic analysis can provide $3 \mathrm{D}$ structure at atomic resolution, the solved structure of G-quadruplex may be different from that present in aqueous solution. ${ }^{12}$ The Gquadruplex structure can also be topologically detected by circular dichroism (CD) analysis. ${ }^{13}$ Fluorescence emission and fluorescence resonance energy transfer (FRET) could be applied for the study of the G-quadruplex structures in solution; ${ }^{14-16}$ however, any factor that affects static and dynamic quenching can cause their changes, and thus far, the detection specificity is limited. ${ }^{17}$

Fluorescence anisotropy (FA) is a popular analytical technology that has been widely used in clinical analysis, food analysis, and environmental monitoring. FA is a real-time and truly homogeneous technique without the need for separation of bound and free species. Moreover, it is insensitive to the photobleaching of the fluorophore due to its ratiometric property. FA has been applied to immunoassays, single nucleotide polymorphism analysis, DNA-protein interaction assays, protein/peptide-ligand binding assays, and enzyme assays. ${ }^{18,19} \mathrm{FA}$ is also applied for nucleic acid aptamer-based affinity analysis for the detection of small molecules, proteins, and cells. Since the binding of small molecules to fluorescently labeled aptamers only induce a small change in FA, indirect FA methods are preferentially developed, e.g., competitive displace-

Received: August 12, 2012

Accepted: August 15, 2012

Published: August 15, 2012 
ment, enzymatic cleavage protection, or mass amplification. $^{20-26}$ Without the need for the change in molecular size or molecular weight, FA can be used to detect conformational change of $\alpha$-lactalbumin protein between the initial unfolded state and the molten globule state. ${ }^{27}$ The applications of aptamers for protein and cellular analysis may benefit from their small sizes and from their advantages of ease-of-synthesis and -modification, as compared to antibody. ${ }^{28-33}$ In general, the binding of fluorescent aptamers to target protein may induce a significant increase in FA due to their relatively small size. Just recently, with an exception, we found that the fluorescently labeled anti-human- $\alpha$-thrombin aptamer TA29 when bound to its target protein may generate a large reduction in $\mathrm{FA}^{34,35}$ Interestingly, by taking advantage of such a reduction in FA, a highly sensitive protein signaling assay could be established with dramatically improved specificity. ${ }^{35}$ By the developed assay, human $\alpha$-thrombin spiked in diluted serum can be detected as low as $250 \mathrm{pM}$. In contrast, in a traditional molecular size-dependent FA assay, the thrombin spiked in diluted serum cannot induce reliable FA change even at a 256fold higher concentration. ${ }^{35}$ However, the protein-aptamer systems exploited in these works are pretty complicated and cannot facilitate the study of the underlying methodological principle, by the understanding of which more analytical applications of the developed assays will be brought up.

In this work, we not only extend the previous work to the detection of G-quadruplex structure but also clarify the underlying methodological principle using fluorescence lifetime analysis, and by taking advantage of the involvement of only single-stranded DNA, we highlight the contribution of the fluorescence lifetime change to the development of sensitive fluorescence anisotropy assays. On the basis of fluorescence lifetime change caused by the photoinduced transfer mechanism, we for the first time propose a novel FA approach for sensitively profiling the formation and stability of G-quadruplex structures.

\section{EXPERIMENTAL SECTION}

Materials and Reagents. All oligonucleotides (see Table S1, Supporting Information) were purchased from Sangon Biological Engineering Technology and Services (Shanghai, China), which were fluorescently labeled with the tetramethylrhodamine (TMR) fluorophore and purified by HPLC. The molar concentration of these oligonucleotides was stated as single-strand form unless otherwise noted. The oligonucleotides were prepared in the $10 \mathrm{mM}$ Tris- $\mathrm{HCl}$ buffer ( $\mathrm{pH}$ 7.5), and the oligonucleotide concentration was quantified by measuring the absorbance at $260 \mathrm{~nm}$ using Nanodrop 2000 (Thermo Scientific, Wilmington, DE). Other reagents used in the experiment are of analytical grade and supplied by Amresco (Solon, $\mathrm{OH}$ ) or Beijing Chemical Reagents (Beijing, China). Ultrapure water for preparing solution was obtained through a Purelab Ultra Elga Labwater system (VWS Ltd., High Wycombe, Bucks HP14 3JH, UK) with an electrical resistivity of $18.2 \mathrm{M} \Omega \cdot \mathrm{cm}$.

Fluorescence Anisotropy Analysis. All fluorescence measurements were performed on a Fluoromax- 4 spectrometer (Horiba Jobin Yvon Inc. Edison, NJ) at room temperature. The stock solutions of oligonucleotides $(20 \mathrm{nM})$ were prepared in the buffer of $10 \mathrm{mM}$ Tris- $\mathrm{HCl}$ ( $\mathrm{pH} 7.5)$ plus $\mathrm{KCl}$ with a varying concentration. Quartz cuvettes with a volume of $150 \mu \mathrm{L}$ were used throughout the measurement. The fluorescence emission spectra of the oligonucleotides were recorded from
570 to $650 \mathrm{~nm}$ with excitation at $555 \mathrm{~nm}$. Both the excitation and emission slits were set at $5 \mathrm{~nm}$, and the scan rate was 300 $\mathrm{nm} / \mathrm{min}$. The fluorescence anisotropy of the oligonucleotides was measured with excitation at $555 \mathrm{~nm}$ and emission at 580 $\mathrm{nm}$. Slits for the excitation and the emission were set at 5 and $10 \mathrm{~nm}$, respectively. The integration time was set at $0.5 \mathrm{~s}$. Fluorescence anisotropy was measured using the L-format configuration. The fluorescence anisotropy $(r)$ was defined as:

$$
r=\left(I_{\mathrm{VV}}-G \times I_{\mathrm{VH}}\right) /\left(I_{\mathrm{VV}}+2 G \times I_{\mathrm{VH}}\right)
$$

and

$$
G=I_{\mathrm{HV}} / I_{\mathrm{HH}}
$$

where the subscripts $V$ and $H$ refer to the vertical and horizontal orientation of the polarizer. The first subscript indicates the position of the excitation polarizer, and the second is for the emission polarizer. $G$ is the grating factor of the spectrofluorometer, which is used to correct for the wavelength response to polarization of the emission optics and detectors. Three data points were collected for each fluorescence anisotropy measurement, and the averaged value was used for further data processing.

Fluorescence Lifetime Analysis. The fluorescence lifetime of the probe was measured on home-built time correlated single photon counting (TCSPC) equipment. In the TCSPC experiment, the output of an OPA pumped by a $1 \mathrm{kHz}$ amplified Ti:sapphire laser (Spectral Physics, Mountain View, $\mathrm{CA})$ was used as the excitation source (538 nm, $100 \mathrm{fs})$. A single lens was used to focus the excitation beam into the sample, and the subsequent fluorescence was collected in a back scattering geometry with a parabolic mirror. The emission was sent to a monochromator (SP2358, Princeton Instruments, Acton, MA) and detected using a single photon avalanche diode (PDM 50CT, Micro Photon Devices, Bolzano, Italy). The nuclear instrumentation module (NIM) timing-output of the PDM 50CT and the output of a fast photodiode (TDA 200, PicoQuant GmbH, Berlin, Germany) were, respectively, connected to a TimeHarp 200 correlator (PicoQuant GmbH, Berlin, Germany) as the start pulse and stop pulse. Magic angle detection was used to avoid the effect of TMR reorientation. The instrument response function of this apparatus was about 200 ps.

Assuming no other processes result in loss of anisotropy, the expected relationship of anisotropy and lifetime is described by the Perrin equation: ${ }^{36}$

$$
\begin{aligned}
& r=\frac{r_{0}}{1+(\tau / \theta)} \\
& \theta=\eta V / R T \\
& \text { so } \quad \frac{1}{r}=\frac{1}{r_{0}}+\frac{\tau R T}{r_{0} \eta V}
\end{aligned}
$$

where $r_{0}$ is the anisotropy that would be measured in the absence of rotational diffusion, $\theta$ is the rotational correlation time for the diffusion process, $\tau$ is lifetime, $R$ is the gas constant, $T$ is the temperature in (K), $\eta$ is the viscosity, and $V$ is the molecular volume.

Circular Dichroism (CD) Measurement. All the CD spectra measurements were conducted on a Jasco J-815 CD spectrometer (Tokyo, Japan) at room temperature. In CD analysis, oligonucleotides $(2.5-3.0 \mu \mathrm{M})$ were titrated with different concentrations of $\mathrm{KCl}$ in the buffer of $10 \mathrm{mM}$ Tris- 
$\mathrm{HCl}$ ( $\mathrm{pH} 7.5$ ). Cuvettes with $10-\mathrm{mm}$ path length were used. The CD spectra were recorded from 220 to 320 or $350 \mathrm{~nm}$ and were normalized by subtraction of the background from the blank and averaged over triplicate measurements.

Atomistic Molecular Dynamic Simulation. All molecular dynamic (MD) simulations were carried out using the AMBER 10 package $^{37}$ with the parm $99 \mathrm{SB}^{38}$ and parmbsc0 ${ }^{39}$ force field parameters. Each system was solvated with TIP3P water. ${ }^{40}$ The DNA strands were immersed in a rectangular water box $12.0 \AA$. Langevin dynamics was used to maintain the temperatures $(300-400 \mathrm{~K})$ with a collision frequency of $1.0 \mathrm{ps}$. The integration time step was set to $0.002 \mathrm{ps}$, and the nonbonded interaction cutoff distance was $10 \AA$.

\section{RESULTS AND DISCUSSION}

Association of a Large Reduction in FA with the Formation of G-Quadruplex Structure. We first examined one known G-quadruplex motif, 15 mer thrombin binding aptamer (TA15) with a sequence of 5'-GGT TGG TGT GGT TGG-3'. ${ }^{5}$ By forming G-quadruplex structure, ${ }^{5,6}$ TA15 binds to human $\alpha$-thrombin, a central enzyme of blood coagulation, and inhibits its fibrin-clotting activity. A single fluorophore, tetramethylrhodamine (TMR), was internally labeled at the seventh thymine of TA15 (T7-TMR-TA15), allowing for sensitive FA measurement. It was observed that the FA value of T7-TMR-TA15 (Figure 1A) gradually decreases with the increasing concentration of $\mathrm{K}^{+}(0-250 \mathrm{mM})$. The maximum reduction in FA of T7-TMR-TA15 $(\Delta r=-0.059)$ was obtained in the presence of $100-250 \mathrm{mM} \mathrm{K}^{+}$. Since $\mathrm{K}^{+}$can
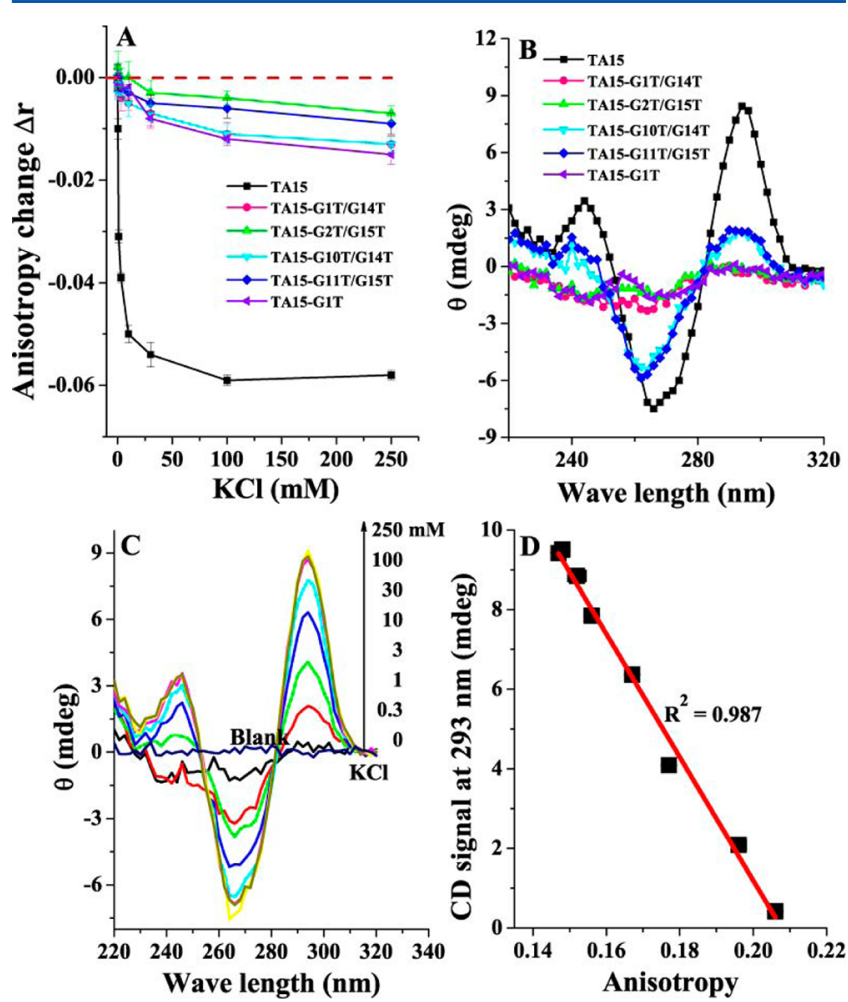

Figure 1. (A) The fluorescence anisotropy change $(\Delta r)$ of T7-TMRTA15 and its mutants in the presence of $\mathrm{KCl}$. (B) CD spectra of T7TMR-TA15 and its mutants at $250 \mathrm{mM} \mathrm{KCl}$. (C) CD spectra of T7TMR-TA15 titrated with KCl. (D) The plot of CD signal at $293 \mathrm{~nm}$ against the fluorescence anisotropy value for T7-TMR-TA15. The error bars represent the $\mathrm{SD}$ of the triplicate measurements. effectively promote TA15 to form a G-quadruplex structure, ${ }^{41-43}$ it is reasonable to believe that the observed reduction in FA is associated with the formation of the G-quadruplex structure.

The formation of G-quadruplex structure was confirmed using the well-known CD analysis (Figure 1B); however, CD analysis requires one-thousand times higher concentration of the oligonucleotide probe $(2.5 \mu \mathrm{M})$ than the FA assay (down to $2.0 \mathrm{nM}$ ). The CD spectra of T7-TMR-TA15 display a peak at $293 \mathrm{~nm}$ and a valley at $263 \mathrm{~nm}$ (Figure 1B,C), which is characteristic of an antiparallel G-quadruplex structure. ${ }^{41-46}$ The amplitude of characteristic CD signal (at $293 \mathrm{~nm}$ ) also increases with the increasing concentration of $\mathrm{K}^{+}$, and the maximum was obtained at $100-250 \mathrm{mM} \mathrm{K}^{+}$. The increased characteristic $\mathrm{CD}$ signal indicates more G-quadruplex formed or the enhanced stability of G-quadruplex structure. ${ }^{17,42}$ Interestingly, the reduction in FA is inversely proportional to the increase in CD signal at $293 \mathrm{~nm}$ (Figure 1D). The good correlation between FA reduction and $\mathrm{CD}$ signal increase in characteristic peak $\left(R^{2}=0.987\right)$ supports the correlation of the large reduction in FA with the formation of G-quadruplex structure.

To test whether the reduction in FA is specific for the Gquadruplex formation, we further examined five mutant TA15 probes, which were obtained by substitution of one or double G with $\mathrm{T}$ (G1T, G1T/G14T, G2T/G15T, G10T/G14T, G11T/G15T; see Table S1, Supporting Information). These probes were labeled at the seventh thymine as well. Compared with T7-TMR-TA15, these mutant probes cannot fold into stable G-quadruplex structure in the presence of $\mathrm{K}^{+}$(Figure 1B) and just have slight decrease in FA $\left(-0.014 \leq \Delta r_{\max }<0\right.$, Figure $1 A)$, showing that the large reduction in FA is specifically associated with G-quadruplex formation.

Mechanism of the Reduction in FA. The next question is how the formation of G-quadruplex structure can induce a large reduction in FA. It is known to us that FA allows the observation of rotational motions of the fluorophore functionalized object during the lifetime of the excited state, which depends on the molecular size and lifetime at a constant temperature and solution viscosity according to the Perrin equation (see Fluorescence Lifetime Analysis). Herein, the reduction in FA should not be attributed to the change in molecular size of complex because the molecular weight of $\mathrm{K}^{+}$ $(M W=39)$ is negligible compared to that of the aptamer probe $(\mathrm{MW}=5291)$. The binding of the $\mathrm{K}^{+}$to $\mathrm{T} 7-\mathrm{TMR}-\mathrm{TA} 15$ would only change $0.7 \%$ of the molecular mass, which is impossible to significantly slow down the rotation of the labeled fluorohore and to produce a large FA change by molecular mass. Therefore, it is suggested that the reduction in FA of T7TMR-TA15 induced by $\mathrm{K}^{+}$may result from the fluorescence lifetime change upon formation of G-quadruplex, which may be caused by the alteration in the intramolecular interactions of the labeled fluorophore with the G-quadruplex motif. The mechanism for the reduction in FA was investigated in detail using molecular dynamic simulation, fluorescence intensity and spectra analysis, and time-resolved fluorescence for lifetime measurement.

To investigate the intramolecular interactions, we first performed three independent atomistic molecular dynamic (MD) simulations (traj_1, traj_2, and traj_3) on T7-TMRTA15 in explicit solvent. In the unfolded state, two strong interactions of the labeled TMR with stacked T4-G5-G6 (TMR-T4-G5-G6 stacking, Figure 1A) and with G2 (TMR-G2 
A)

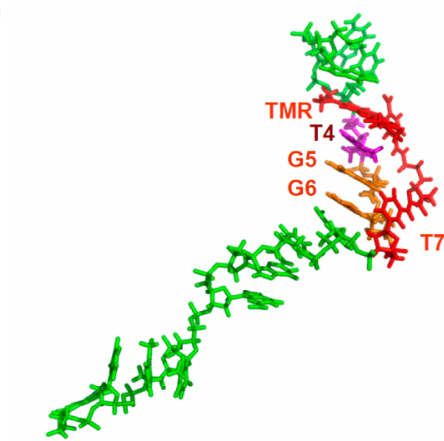

C)

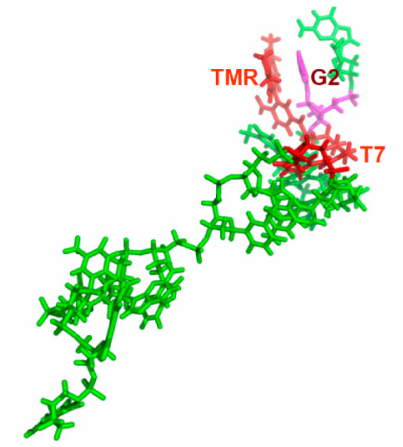

B)

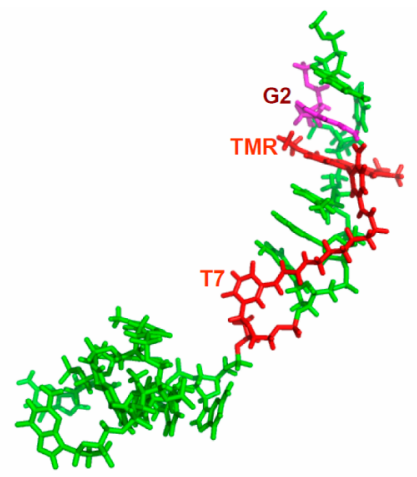

D)

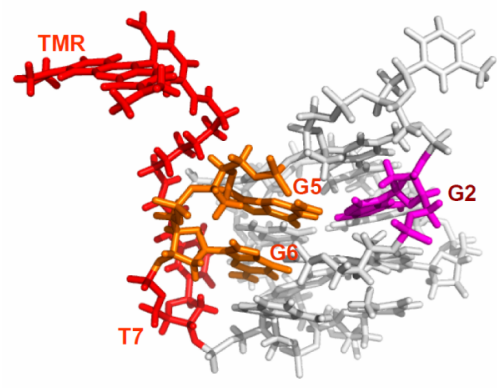

Figure 2. Representative conformations of the unfolded and folded states of T7-TMR-TA15 were obtained from three independent atomistic MD simulations. In the unfolded state, (A) traj_1, TMR-T4-G5-G6 stacking; (B) traj_2, TMR-G2 stacking, and (C) traj_3, TMR-G2 stacking. In folded state (D), TMR was released from the formed G-quadruplex structure and the intramolecular interactions of the labeled TMR with adjacent bases were diminished.

stacking, Figure 2B,C) were observed. As revealed by the traj_1 simulation, the TMR-T4-G5-G6 stacking is very stable (Figure $2 \mathrm{~A}$ ), implying that $\mathrm{T} 4$ may play a role in stabilizing the intramolecular interactions in the unfolded state of T7-TMRTA15; more specifically, TMR could interact with G5/G6 indirectly via the stacking interaction (TMR-T4-G5-G6). When the G-quadruplex structure forms, the TMR-T4 stacking disappears (Figure 2D).

As simulated by other two trajectories (traj_2 and traj_3), the possibilities of strong TMR-G2 interaction mode may be greater than that of TMR-T4-G5-G6 mode in the unfolded state (Figure 2B,C). Similarly, the strong TMR-G2 interaction is diminished when G-quadruplex structure forms (Figures S1 and S2, Supporting Information).

As revealed by $\mathrm{MD}$ simulation, prior to the formation of Gquadruplex structure, the intramolecular interactions between the labeled fluorophore and adjacent guanine bases can occur. It is known that these intramolecular interactions can cause fluorescence quenching through the photoinduced electron transfer mechanism. ${ }^{47-49}$ Therefore, the fluorescence should be restored when these interactions are eliminated upon the formation of highly ordered structure. Indeed, the fluorescence signal of T7-TMR-TA15 increases upon addition of $\mathrm{K}^{+}$to form G-quadruplex (see Figure S3, Supporting Information). These results confirm the occurrence of the intramolecular interactions between the labeled TMR and guanine bases and their elimination upon the G-quadruplex formation.

We further determined the lifetime of T7-TMR-TA15 to test whether the lifetime changes upon the formation of Gquadruplex. Figure 3 shows the fluorescence decay curves of T7-TMR-TA15 (Figure 3A) and the mutant probe T7-TMRTA15-G1T (Figure 3B) in the absence and presence of 250 $\mathrm{mM} \mathrm{KCl}$, respectively. The average fluorescence lifetime data obtained with exponential fit of the fluorescence decay curves were listed in Table 1. Upon addition of G-quadruplexinducible $\mathrm{K}^{+}$, the average fluorescence lifetime $(\tau)$ of T7-TMRTA15 increases from 2.31 to $2.87 \mathrm{~ns}$, indicating a significant fluorescence lifetime change of the labeled TMR upon the formation of G-quadruplex structure and supporting the elimination of the intramolecular interactions between the labeled TMR and the adjacent G bases. The result is consistent with previous reports showing that the lifetime of TMR increases when the intramolecular interaction between TMR and guanine was eliminated. ${ }^{50-52}$ According to the eq 5 (Fluorescence Lifetime Analysis), the fluorescence anisotropy $(r)$ is reversely proportional to the fluorescence lifetime $(\tau)$. Therefore, the observed increase of lifetime should contribute to the reduction in FA upon the formation of G-quadruplex structure. In contrast, to the mutant probe T7-TMR-TA15G1T, the fluorescence lifetime almost did not change after addition of high concentration of $\mathrm{KCl}$ (Figure $3 \mathrm{~B}$ and Table 1); meanwhile, the corresponding FA response was just slightly reduced (Figure 1A).

Illustration of the FA Approach for Detection of GQuadruplex Structure. On the basis of the above results, a summary of the FA approach for probing G-quadruplex structures was illustrated as Scheme 1. Basically, a fluorophore, selectively labeled in the G-quadruplex motif, may intramolecularly interact with the adjacent $\mathrm{G}$ bases in the randomly coiled state. ${ }^{47,48,53,54}$ The intramolecular interaction might restrict the local rotation of the labeled fluorophore; meanwhile, the concomitant photoinduced electron transfer (PET) could occur between the selectively labeled fluorophore and the adjacent $\mathrm{G}$ bases, rendering a short fluorescence lifetime. Both the restricted local rotation and short fluorescence lifetime of the fluorophore would increase its fluorescence anisotropy value. When G-quadruplex motif folds into a high order of structural architecture from the random coil state, it weakens 

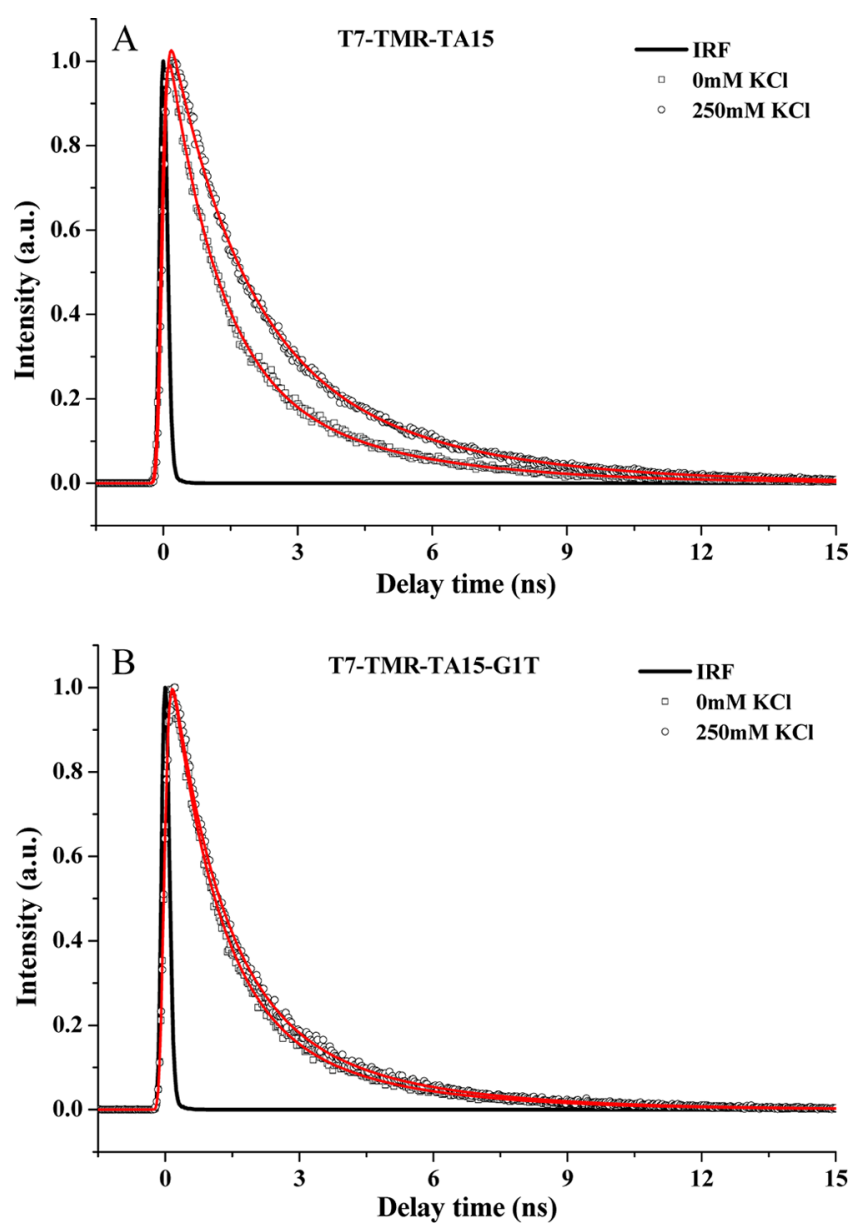

Figure 3. Fluorescence decay time profiles of T7-TMR-TA15 (A) and its mutant T7-TMR-TA15-G1T (B) in the absence and presence of $250 \mathrm{mM} \mathrm{KCl}$ obtained by the TCSPC measurement. The red curves are the fitted curves.

Table 1. Fluorescence Lifetime $(\tau)$ of T7-TMR-TA15 and T7-TMR-TA15-G1T Obtained with Exponential Fit of the Fluorescence Decay Curve

\begin{tabular}{lccccc}
\multicolumn{1}{c}{ sample } & $\begin{array}{c}\tau_{1} \\
(\mathrm{~ns})\end{array}$ & $A_{1}$ & $\begin{array}{c}\tau_{2} \\
(\mathrm{~ns})\end{array}$ & $A_{2}$ & $\begin{array}{c}\text { average } \tau \\
(\mathrm{ns})\end{array}$ \\
T7-TMR-TA15 & 1.05 & 0.45 & 3.35 & 0.55 & 2.31 \\
T7-TMR-TA15 + 250 mM & 1.50 & 0.39 & 3.75 & 0.61 & 2.87 \\
$\quad$ KCl & & & & & \\
T7-TMR-TA15-G1T & 1.00 & 0.50 & 3.05 & 0.5 & 2.02 \\
T7-TMR-TA15-G1T +250 & 1.05 & 0.47 & 3.20 & 0.53 & 2.19 \\
$\quad$ mM KCl & & & & \\
Note: $\tau=\left(A_{1} \cdot \tau_{1}+A_{2} \cdot \tau_{2}\right) /\left(A_{1}+A_{2}\right)$. & & &
\end{tabular}

the intramolecular interaction between the fluorophore and the adjacent guanine bases. Consequently, the fluorophore is released out of the interacting region and the relaxed fluorophore can rotate faster. Because FA is a measure of the diffusional rotation of a fluorophore and displays low response to the fast rotation, ${ }^{36}$ it will lead to a specific reduction in FA (Scheme 1). Accompanied with the elimination of PET, the fluorescence lifetime becomes longer, further reducing the fluorescence anisotropy response. ${ }^{50-52}$ Therefore, the formation of G-quadruplex structures can be specifically manifested by a large reduction in FA.

Applications. In addition to T7-TMR-TA15, three probes of TA15 with a single TMR labeled at T9, T12, or T13 display
Scheme 1. Schematic Illustration of Fluorescence Anisotropy Reduction of the Fluorescently Labeled Oligonucleotide upon G-Quadruplex Formation

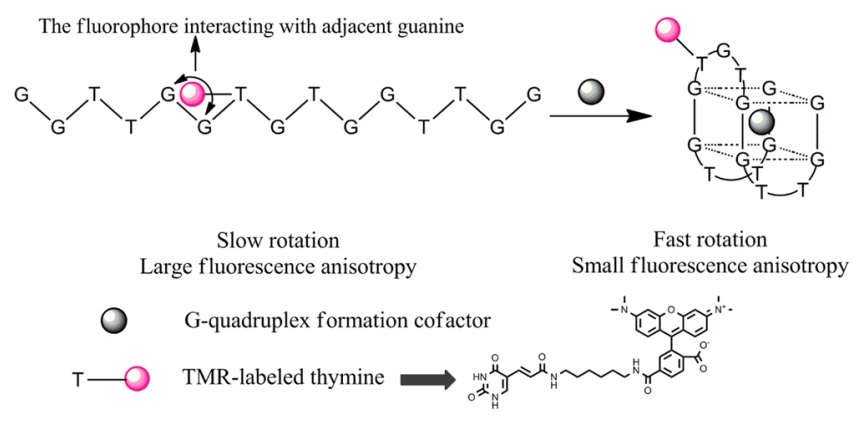

a significant reduction in FA upon the formation of Gquadruplex (Figure S4, Supporting Information). The reduction in FA is well correlated with the increase in typical CD signal of G-quadruplex structure at $293 \mathrm{~nm}\left(R^{2}=0.90-0.99\right)$ (Figure S5, Supporting Information). Therefore, T9, T12, and T13 can also be identified as FA-sensitive sites of TA15 in response to the G-quadruplex formation. The maximum reduction in FA response is obtained by labeling TA15 at T7. However, the other two thymine sites (T3 and T4) of TA15 are not FA responsible (Figure S4, Supporting Information). The reason is not clear yet.

This FA approach is applicable to the investigation of the interactions between TA15 and small cationic ligands. In addition to $\mathrm{K}^{+}$, other cations, e.g, $\mathrm{NH}_{4}^{+}, \mathrm{Na}^{+}, \mathrm{Mg}^{2+}, \mathrm{Ba}^{2+}$, and $\mathrm{Sr}^{2+}$, which can induce TA15 to form G-quadruplex with varying stability as shown by $\mathrm{CD}$ analysis (Figure S6, Supporting Information), also similarly reduce the FA values of T7-TMR-TA15. The reduction in FA of T7-TMR-TA15 is inversely proportional to the amplitude of its characteristic CD bands (Figure 4). The good linear correlation $\left(R^{2}=0.996\right)$

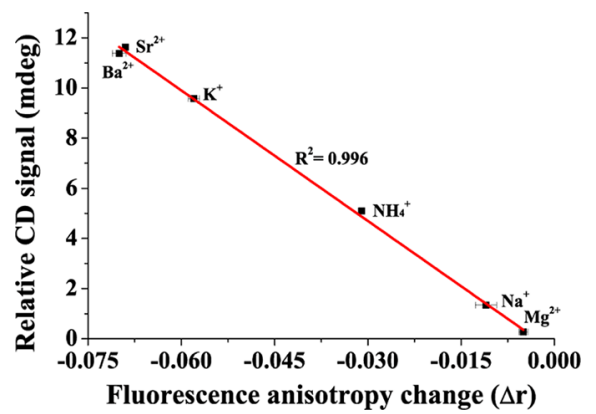

Figure 4. Linear correlation of the $\mathrm{CD}$ amplitude at the corresponding maximum absorption $\left(293 \mathrm{~nm}\right.$ for $\mathrm{Na}^{+}, \mathrm{Mg}^{2+}, \mathrm{NH}_{4}{ }^{+}$, and $\mathrm{K}^{+}$and 303 $\mathrm{nm}$ for $\mathrm{Ba}^{2+}$ and $\left.\mathrm{Sr}^{2+}\right)$ with the fluorescence anisotropy change $(\Delta r)$ of T7-TMR-TA15 in the presence of different cations $(250 \mathrm{mM} \mathrm{KCl}, 250$ $\mathrm{mM} \mathrm{NaCl}, 250 \mathrm{mM} \mathrm{NH} \mathrm{H}_{4} \mathrm{Cl}, 30 \mathrm{mM} \mathrm{MgCl} 2,100 \mu \mathrm{M} \mathrm{BaCl}_{2}$, and 100 $\mu \mathrm{M} \mathrm{SrCl} 2$ ).

suggests that reduction in FA can be used to evaluate the capability of small ligands to induce the G-quadruplex formation, showing the possibility the screening of ligands, which may promote G-quadruplex formation and become candidates for cancer therapies.

It was observed that two divalent cations $\mathrm{Ba}^{2+}$ and $\mathrm{Sr}^{2+}$ can induce TA15 to form stable G-quadruplex structure at very low concentration $(100 \mu \mathrm{M})$, which is a hundred times lower than the concentration involved by the other four cations. 
Interestingly, the two cations can induce a large reduction in FA $(\Delta r=\sim 0.070$, Figure 3$)$ at the same low concentration (100 $\mu \mathrm{M})$. These results may indicate that the reduction in FA is very sensitive to the occurrence of the interactions between Gquadruplex motifs and small ligands by monitoring the formation of G-quadruplex structures.

In this work, most importantly, it is established that the large reduction in FA is specifically associated with the unfolding/ folding transition of G-quadruplexes. Conversely, the binding events (e.g., protein-DNA binding) often render an increase in fluorescence anisotropy due to an increase in molecular size of the complex if no G-quadruplex forms. ${ }^{55-57}$ Compared with FRET assays, ${ }^{14,16}$ which require careful choice of donor/ acceptor pair, FA assays have pronounced advantages of high specificity, single labeling, and insensitivity to the variance in fluorescence intensity. ${ }^{55,56}$

\section{CONCLUSIONS}

In summary, we demonstrate a fluorescence anisotropy approach for the study of G-quadruplex structures and their interactions with small ligands. The approach is examined and validated using one normal G-quadruplex motifs, five mutated motifs, and six small ligands. Fluorescence emission spectroscopy, $\mathrm{CD}$ spectroscopy, and $\mathrm{MD}$ simulations confirmed the underlying mechanism and the presented approach. Moreover, we show that FA-sensitive sites located in G-quadruplex motifs can be identified by single labeling. Following the photoinduced electron transfer mechanism, this study may indicate the possibility of the development and design of fluorescence anisotropy assays for the detection of proteins and DNA in general purpose.

\section{ASSOCIATED CONTENT}

\section{S Supporting Information}

Additional information as noted in text. This material is available free of charge via the Internet at http://pubs.acs.org.

\section{AUTHOR INFORMATION}

\section{Corresponding Author}

*Telephone/Fax: 86-10-62849600. E-mail: hlwang@rcees.ac. cn.

\section{Notes}

The authors declare no competing financial interest.

\section{ACKNOWLEDGMENTS}

This work was supported by the grants from the National Basic Research Program of China (2011CB936001 and 2009CB421065) and the National Natural Science Foundation of China (21077129, 20890112, 21125523, and 20921063). We thank Dr. Lianghong Guo for his support in CD analysis. G.H.L. designed the strategy and protocols for the theoretical modeling. H.J.S. performed the detailed modeling study. G.H.L. and H.J.S. analyzed the MD data.

\section{REFERENCES}

(1) Simonsson, T. Biol. Chem. 2001, 382, 621.

(2) Davis, J. T. Angew. Chem., Int. Ed. 2004, 43, 668.

(3) Georgiades, S. N.; Karim, N. H. A.; Suntharalingam, K.; Vilar, R. Angew. Chem., Int. Ed. 2010, 49, 4020.

(4) Kairala, D.; Dhakal, S.; Ashbridge, B.; Sannohe, Y.; Rodriguez, R.; Sugiyama, H.; Balasubramanian, S.; Mao, H. Nat. Chem. 2011, 3, 782.

(5) Bock, L. C.; Griffin, L. C.; Latham, J. A.; Vermass, E. H.; Toole, J. J. Nature 1992, 355, 564.
(6) Tasset, D. M.; Kubik, M. F.; Steiner, W. J. Mol. Biol. 1997, 272, 688

(7) Liu, J.; Cao, Z.; Lu, Y. Chem. Rev. 2009, 109, 1948.

(8) Krishnam, Y.; Simmel, F. C. Angew. Chem., Int. Ed. 2011, 50, 3124.

(9) Wang, Y.; Patel, D. J. Structure 1993, 1, 263.

(10) Qu, N. Q.; Lim, K. W.; Teo, M. H.; Heddi, B.; Phan, A. T. Nucleic Acids Res. 2011, 39, 9448.

(11) Parkinson, G. N.; Lee, M. P.; Neidle, S. Nature 2002, 417, 876.

(12) Li, J.; Correia, J. J.; Wang, L.; Trent, J. O.; Chaires, J. B. Nucleic Acids Res. 2005, 33, 4649.

(13) Dapic, V.; Abdomerovic, V.; Marrington, R.; Peberdy, J.; Rodger, A.; Trent, J. O.; Bate, P. J. Nucleic Acids Res. 2003, 31, 2097.

(14) Ueyama, H.; Takagi, M.; Takenaka, S. J. Am. Chem. Soc. 2002, $124,14286$.

(15) Juskowiak, B. Anal. Chim. Acta 2006, 568, 171.

(16) Takenaka, S.; Juskowiak, B. Anal. Sci. 2011, 27, 1167.

(17) Lane, A. N.; Chaires, J. B.; Gray, R. D.; Trent, J. O. Nucleic Acids Res. 2008, 36, 5482.

(18) Checovich, W. J.; Bolger, R. E.; Burke, T. Nature 1995, 375, 254.

(19) Jameson, D. M.; Ross, J. A. Chem. Rev. 2010, 110, 2685.

(20) Cruz-Aguado, J. A.; Penner, G. Anal. Chem. 2008, 80, 8853.

(21) Ruta, J.; Perrier, S.; Ravelet, C.; Fize, J.; Peyrin, E. Anal. Chem. 2009, 81, 7468

(22) Perrier, S.; Ravelet, C.; Guieu, V.; Fize, J.; Roy, B.; Perigaud, C.; Peyrin, E. Biosens. Bioelectron. 2010, 25, 1652.

(23) Kidd, A; Guieeu, V.; Perrier, S.; Ravelet, C.; Peyrin, E. Anal. Bioanal. Chem. 2011, 401, 3229.

(24) Zhu, Z.; Schmidt, T.; Mahrous, M.; Guieu, V.; Perrier, S.; Ravelet, C.; Peyrin, E. Anal. Chim. Acta 2011, 707, 191.

(25) Cui, L.; Zou, Y.; Lin, N.; Zhu, Z.; Jenkins, G.; Yang, C. J. Anal. Chem. 2012, 84, 5535.

(26) Huang, Y.; Zhao, S.; Chen, Z.-F.; Shi, M.; Liang, H. Chem. Commun. 2012, 48, 7480.

(27) Canet, D.; Doering, K.; Dobson, C. M.; Dupont, Y. Biophys. J. 2001, 80, 1996.

(28) Potyrailo, R. A.; Conrad, R. C.; Ellington, A. D.; Hieftje, G. M. Anal. Chem. 1998, 70, 3419.

(29) Fang, X.; Cao, Z.; Beck, T.; Tan, W. Anal. Chem. 2001, 73, 5752.

(30) McCauley, T. G.; Hamaguchi, N.; Stanton, M. Anal. Biochem. 2003, 319, 244.

(31) Gokulrangan, G.; Unruh, J. R.; Holub, D. F.; Ingram, B.; Johnson, C. K.; Wilson, G. S. Anal. Chem. 2005, 77, 1963-1970.

(32) Deng, T.; Li, J.; Zhang, L.-L.; Jiang, J.-H.; Chen, J.-N.; Shen, G.L.; Yu, R.-Q. Biosens. Bioelectron. 2010, 25, 1587.

(33) Zou, M.; Chen, Y.; Xu, X.; Huang, H.; Liu, F.; Li, N. Biosens. Bioelectron. 2012, 32, 148.

(34) Zhang, D.; Lu, M.; Wang, H. J. Am. Chem. Soc. 2011, 133, 9188.

(35) Zhang, D.; Zhao, Q.; Zhao, B.; Wang, H. Anal. Chem. 2012, 84, 3070.

(36) Lakowicz, J. R. Principles of Fluorescence Spectroscopy, 3rd ed.; Springer-Verlag: Berlin Heidelberg, 2006.

(37) Case, D. A.; Darden, T.; Cheatham, III, T. E.; Simmerling, C.; Wang, J.; Duke, R. E.; Luo, R.; Crowley, M.; Walker, R.; Zhang, W.; Merz, K. M.; Wang, B.; Hayik, S.; Roitberg, A.; Seabra, G.; Kolossváry, I.; Wong, K. F.; Paesani, F.; Vanicek, J.; Wu, X.; Brozell, S. R.; Steinbrecher, T.; Gohlke, H.; Yang, L.; Tan, C.; Mongan, J.; Hornak, V.; Cui, G.; Mathews, D. H.; Seetin, M. G.; Sagui, C.; Babin, V.; Kollman, P. A. AMBER 10, 2008.

(38) Hornak, V.; Abel, R.; Okur, A.; Strockbine, B.; Roitberg, A.; Simmerling., C. Proteins 2006, 65, 712.

(39) Pérez, A.; Marchán, I.; Svozil, D.; Sponer, J.; Cheatham, T.; Laughton, C. A.; Orozco, M. Biophys. J. 2007, 92, 3817.

(40) Jorgensen, W. L.; Chandrasekhar, J.; Madura, J. D.; Impey, R. W.; Klein, M. L. J. Chem. Phys. 1983, 79, 926-935.

(41) Li, T.; Wang, E.; Dong, S. Anal. Chem. 2010, 82, 7576.

(42) Kankia, B. I.; Marky, L. A. J. Am. Chem. Soc. 2001, 123, 10799.

(43) Olsen, C. M.; Lee, H. T.; Marky, L. A. J. Phys. Chem. B 2009, $113,2587$. 
(44) Poniková, S.; Antalík, M.; Hianik, T. Gen. Physiol. Biophys. 2008, 27, 271.

(45) Nagatoishi, S.; Tanaka, Y.; Tsumoto, K. Biochem. Biophys. Res. Commun. 2007, 352, 812.

(46) Lin, P. H.; Chen, R. H.; Lee, C. H.; Chang, Y.; Chen, C. S.; Chen, W. Y. Colloid Surf., B: Biointerfaces 2011, 88, 552.

(47) Nazarenko, I.; Pires, R.; Lowe, B.; Obaidy, M.; Rashtchian, A. Nucleic Acids Res. 2002, 30, 2089.

(48) Torimura, M.; Kurata, S.; Yamada, K.; Yokomaku, T.; Kamagata, Y.; Kanagawa, T.; Kurane, R. Anal. Sci. 2001, 17, 155.

(49) Lewis, F. D.; Letsinger, R. L.; Wasielewski, M. R. Acc. Chem. Res. 2001, 34, 159.

(50) Edman, L.; Mets, U.; Rigler, R. Proc. Natl. Acad. Sci. U.S.A. 1996, 93, 6710.

(51) Xia, S. J.; Hu, P. P.; Li, Y. F.; Huang, C. Z.; Huang, T.; Xiao, G. F. Talanta 2009, 79, 1283.

(52) Li, X.; Zhu, R.; Yu, A.; Zhao, X. S. J. Phys. Chem. B 2011, 115, 6265.

(53) Walter, N. S.; Burke, J. M. RNA 1997, 3, 392.

(54) Piestert, O.; Barsch, H.; Buschmann, V.; Heinlein, T.; Knemeyer, J. P.; Weston, K. D.; Sauer, M. Nano Lett. 2003, 3, 979.

(55) Ruta, J.; Perrier, S.; Ravelet, C.; Fize, J.; Peyrin, E. Anal. Chem. 2009, 81, 7468.

(56) Chen, X.; Levine, L.; Kwok, P. Y. Genome Res. 1999, 9, 492498.

(57) Wang, H.; Lu, M.; Tang, M.-S.; Van Houten, B.; Ross, J. B. A.; Weinfeld, M.; Le, X. C. Proc. Natl. Acad. Sci. U.S.A. 2009, 106, 12849. 